\title{
Resource Allocation for Two-relay Networks with Mixed Relaying Protocol in Line Topology
}

\author{
Woong Cho \\ Department of Computer System Engineering, Jungwon University \\ 85, Munmu-ro, Goesan-eup, Goesan-gun, Chungbuk, South Korea \\ wcho@jwu.ac.kr
}

\begin{abstract}
Relay nodes can be used for extending communication coverage or enhancing system performance by relaying the received signal to another node or providing cooperative diversity gain, respectively. In this paper, we analyze the effect of resource allocation in two-relay networks with mixed relaying protocol. We assume that two relay nodes exist between the source node and destination node, and one relay node adopts decode-andforward $(D F)$ relaying protocol and the other one uses amplify-and-forward $(A F)$ relaying protocol. Differential modulation scheme is applied for all cases to bypass channel estimation. First, we compare the performance of proposed system with the systems only using DF protocol and AF protocol. Then, we investigate the performance of the proposed system by considering the location of relay nodes, i.e., co-located and various location scenarios, depending on the existence of direct link. Finally, the effect of both energy and location in the overall performance is analyzed by assuming that two node are co-located.
\end{abstract}

Keywords: Relay networks, decode-and-forward, amplify-and-forward, resource allocation, SER

\section{Introduction}

Relay networks can help extend communication range and enhance the performance of communication system by creating virtual antenna arrays [1-6]. Resource optimization and its effects on relay networks have been analyzed with various modulation scheme and relaying protocols. Differential modulation scheme can be adopted fast fading channel such as vehicular or railway environment since it reduces hardware complexity by obviating channel estimation [7]. Using differential modulation scheme, optimum energy and location allocations are considered in [8] and [9]. For various relaying protocols, i.e., amplify-and-forward (AF) and decode-and-forward (DF), the optimization factors are investigated in [10-11] for both coherent and differential modulation schemes. Recently, Long Term Evolution-Railway (LTE-R) is considered as the evolution of existing networks such as Global System for Mobile Communications Railways (GSM-R) [12-13], and 3-rd Generation Partnership Projects (3GPP) defines the proximity services (ProSe) which uses relays for data transmission [14]. The performance of relay networks and relay selection problem in railway environments are discussed in [15-16]. In addition, relays are widely accepted to apply in wireless communication systems. However, existing works only consider all relay nodes use AF or DF protocol.

In this paper, we consider mixed relaying protocol in which one relay node and adopts $\mathrm{AF}$ protocol and the other relay node uses DF protocol by assuming two relay links are available. The network topology uses line topology which is similar condition in vehicular scenarios such as railway/road environments. We analyze the performance of proposed system by employing differential modulation. Then, resource allocation for the proposed 
system is investigated depending on existence of direct link. The performance of the system is evaluated using symbol error rate (SER).

\section{System Model}

We consider two-relay networks when an arbitrary number of relay exists in railway environments. Figure1 represents system model for line topology where 1.(a) and 1.(b) represents railway environments and road environments, respectively. Different from the general mobile communication systems, trains or vehicles move through railroad track or road and their locations are traceable. For LTE-R systems, base stations are installed along the railroad and a train goes almost straight within one cell coverage. We denote one source node $s$ which corresponds a base station, two relay nodes $r_{k}, k=1,2$, and one destination node $d$ which corresponds a node located in the train. Similar environments are applied for road environments by replacing a train to a vehicle. We assume that only two relay nodes are used for signal transmission and mixed relying protocol is used. One relay nodes adopts DF relaying protocol which the relay node remodulates the received signal from the source node and forwards it to the destination node, and the other node simply amplify and forward it to the destination node, i.e., AF relaying protocol is applied. We consider differential modulation scheme, in which both relay nodes and destination node bypass channel estimation.

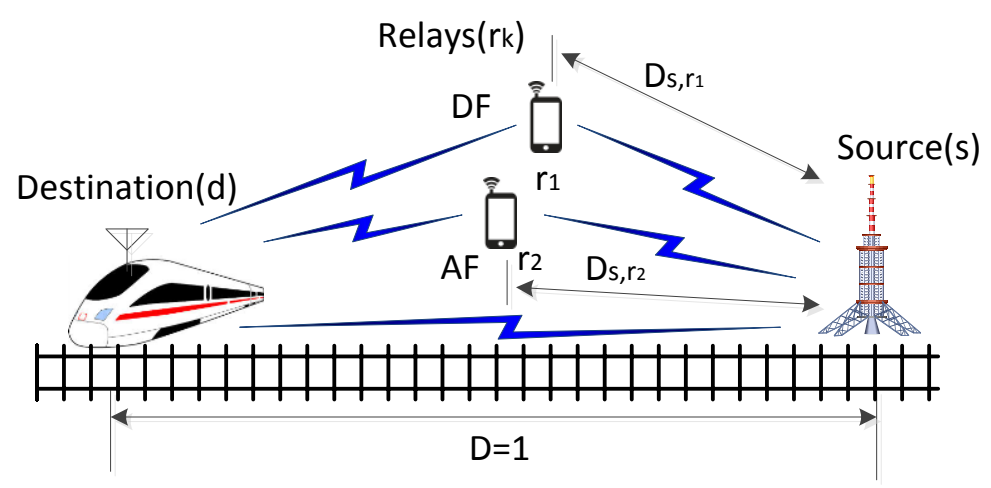

(a)

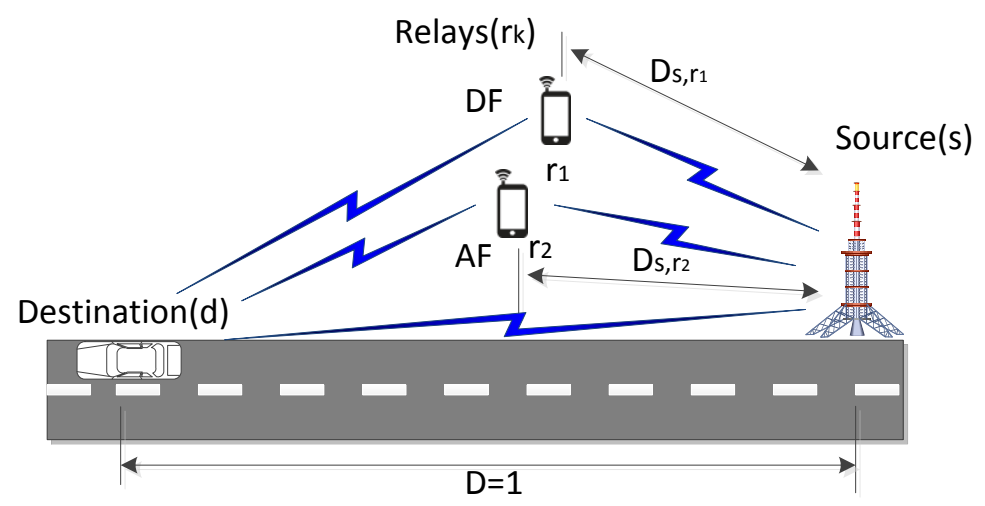

(b)

Figure 1. System Model for Two-relay Network (a) Railway Environments (b) Road Environments

Since we transmit signal using differential scheme, the $\mathrm{n}$-th transmission signal at the source node is $x_{n}^{s}=x_{n-1}^{s} s_{n}$ with $x_{0}^{s}=1$, where $s_{n}=e^{j 2 \pi m_{n} / M}, m_{n} \in\{0,1, \ldots, M-1\}$. Then, 
the received signal at the relay node and destination node are given by equations (1) and (2), respectively.

$$
\begin{gathered}
y_{n}^{s, r_{k}}=\sqrt{\mathrm{E}_{s}} h_{n}^{s, r_{k}} s_{n}+z_{n}^{s, r_{k}}, k=1,2 \\
y_{n}^{s, d}=\sqrt{\mathrm{E}_{s}} h_{n}^{s, d} x_{n}^{s}+z_{n}^{s, d}
\end{gathered}
$$

The received signals from the relay node which apply AF relaying protocol and DF relaying protocol can be represented as equations (3) and (4).

$$
\begin{gathered}
y_{n, A F}^{r_{k}, d}=\sqrt{\mathrm{E}_{r_{k}}} h_{n}^{r_{k}, d} x_{n, A F}^{r_{k}}+z_{n}^{r_{k}, d} \\
y_{n, D F}^{r_{k}, d}=\sqrt{\mathrm{E}_{r_{k}}} h_{n}^{r_{k}, d} x_{n, D F}^{r_{k}}+z_{n}^{r_{k}, d}
\end{gathered}
$$

where, $x_{n, A F}^{r_{k}}=A_{r_{k}} y_{n}^{s, r_{k}}$ with amplification factor $A_{r_{k}}$ is defined as

$$
A_{r_{k}}=\sqrt{\frac{1}{\sigma_{s, r_{k}}^{2} \mathrm{E}_{s}+N_{s, r_{k}}}}
$$

and, remodulated signal $x_{n, D F}^{r_{k}}=x_{n-1, D F}^{r_{k}} \hat{s}_{n}^{r_{k}}, n \geq 1$ with the initial value $x_{n, D F}^{r_{k}}=1, n=0$. In the above equations, $\mathrm{E}_{i}, i \in\left\{s, r_{k}\right\}$ represents energy per symbol at the node $i$, and fading coefficient $h_{n}^{i, j}$ and noise $z_{n}^{i, j}$ are zero-mean complex Gaussian distribution with variance $\sigma_{i, j}^{2}$ and $N_{i, j}, \forall i, j \in\left\{s, r_{k}, d\right\}$, respectively. In this paper, we assume that all fading coefficients are independent. We also assume that all noise components are independent and identically distributed (i.i.d) with $N_{i, j}=N_{0}, \forall i, j \in\left\{s, r_{k}, d\right\}$. Then, we can find instantaneous signal-to-noise ratio (SNR) and the average SNR between the transmitter $i$ and the receiver $j$ as

$$
\gamma_{i, j}=\frac{\left|h_{n}^{i, j}\right|^{2} \mathrm{E}_{i}}{N_{0}}
$$

and

$$
\bar{\gamma}_{i, \mathrm{j}}=\frac{\sigma_{i, j}^{2} \mathrm{E}_{i}}{N_{0}}
$$

for all $i, j \in\left\{s, r_{k}, d\right\}$, respectively.

The destination node demodulates the received by combing signals from the source and two relay nodes. The link which uses AF protocol has complex Gaussian with mean $y_{n-1}^{r_{k}, d} s_{n}$ and variance $2 N_{0}\left(\mathrm{E}_{r_{k}} A_{r_{k}}^{2} \sigma_{r_{k}, d}^{2}+1\right)$. Whereas, link which uses DF protocol has complex Gaussian with mean $y_{n-1}^{r_{k}, d} \hat{s}_{n}$ and variance $2 N_{0}$. Similarly, the direct link has also complex Gaussian with mean $y_{n-1}^{s, d} s_{n}$ and variance $2 N_{0}$. Notice that the mean value of direct signal and relay signal is different although the variance is the same. Then, decision rule for the system with direct link and without direct link are given by 


$$
\begin{aligned}
\hat{\boldsymbol{s}}_{n} & =e^{j 2 \pi m_{n}^{\prime} / M}: m_{n}^{\prime} \\
& =\underset{m \in 0,1, \ldots, M-1}{\arg \max } \mathfrak{R}\left\{\left(y_{n}^{s, d}\right)^{*} y_{n-1}^{s, d} s_{n}+\left(y_{n, A F}^{r_{k}, d}\right)^{*} y_{n-1, A F}^{r_{k}, d} s_{n}+\left(y_{n, D F}^{r_{k}, d}\right)^{*} y_{n-1, D F}^{r_{k}, d} s_{n}\right\}
\end{aligned}
$$

and

$$
\begin{aligned}
\hat{S}_{n} & =e^{j 2 \pi m_{n}^{\prime} / M}: m_{n}^{\prime} \\
& =\underset{m \in 0,1, \ldots, M-1}{\arg \max } \mathfrak{R}\left\{\left(y_{n, A F}^{r_{k}, d}\right)^{*} y_{n-1, A F}^{r_{k}, d} s_{n}+\left(y_{n, D F}^{r_{k}, d}\right)^{*} y_{n-1, D F}^{r_{k}, d} s_{n}\right\}
\end{aligned}
$$

, respectively. In eqs. (8) and (9), $s_{n}=e^{j 2 \pi m / M}$. The only difference of decision rules is the existence of direct link related term, i.e. ${ }^{s, d}$, in equation (8) and (9). To capture the effect of relay location, we defined the relation between variance and relay location as

$$
\sigma_{i, j}^{2}=C \cdot D_{i, j}^{-\alpha}, i, j \in\left\{s, r_{k}, d\right\}
$$

, where $\alpha$ is path loss exponent and we assume $C=1$ and $D_{s, d}=1$.

\section{Simulations}

In this section, we represent simulation results. First, we compare the performance of proposed system with fully AF system and DF system. Figure 2 represents the comparison of performance. In this simulation, we only consider the system without direct link, and $\mathrm{AF}$ and $\mathrm{DF}$ represent two relay nodes use $\mathrm{AF}$ protocol and DF protocol, respectively. In the figure, $x$ axis represent SNR at source and relay node, where we assigned the same SNR in both nodes. The figure shows that the performance is almost the same for all cased in low SNR. However, in high SNR, AF protocol shows best performance and DF protocol has the worst performance. Decoding error at the relay node induces the performance degradation compared with AF protocol. The performance of mixed relaying protocol is located in the middle of $\mathrm{AF}$ and $\mathrm{DF}$ protocol, but diversity gain is the same as the AF protocol.

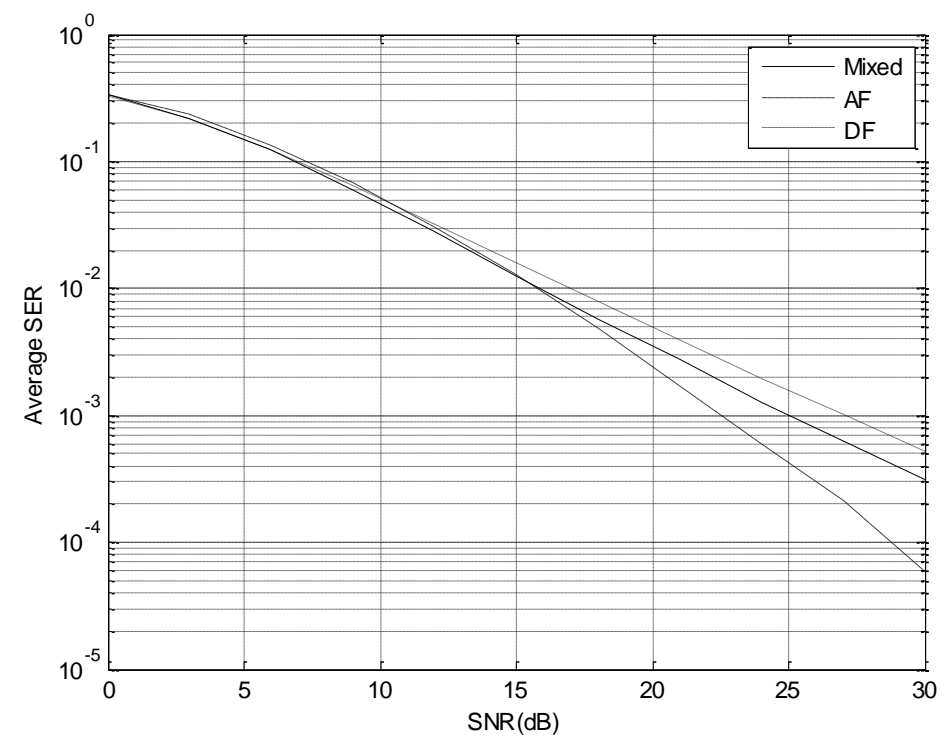

Figure 2. SER Comparison 
Next, we consider various locations of relay nodes in the performance. As we mentioned earlier, by fixing $D_{s, d}=D=1$, we change the location of relay node from 0 to 1 by assuming $D_{s, r}+D_{r, d}=1$.

\subsection{The Effect of Location}

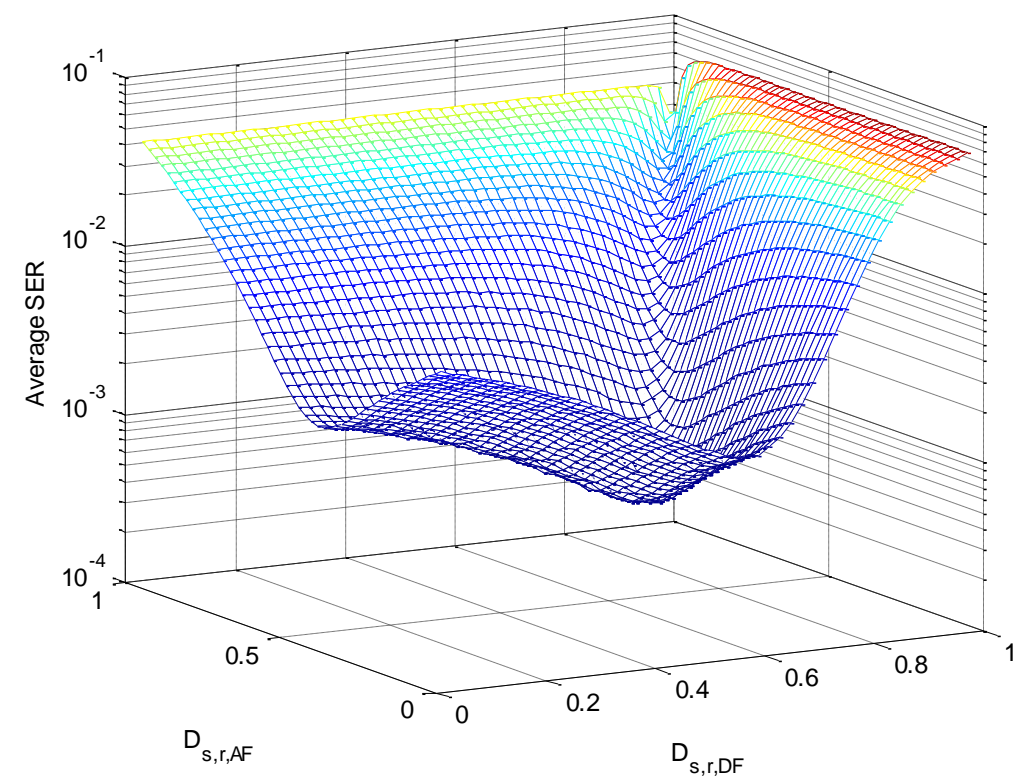

(a)

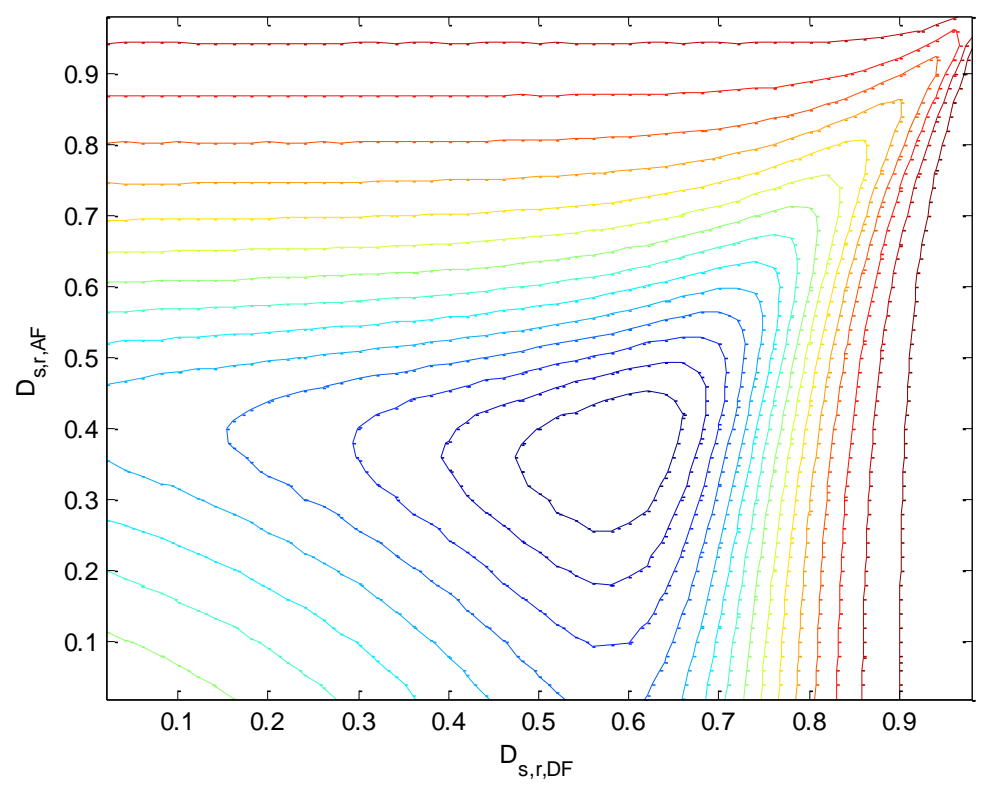

(b)

Figure 3. SER Depending on Various Relay Locations; Systems with Direct Link (a) SER Curve (b) SER Contour 


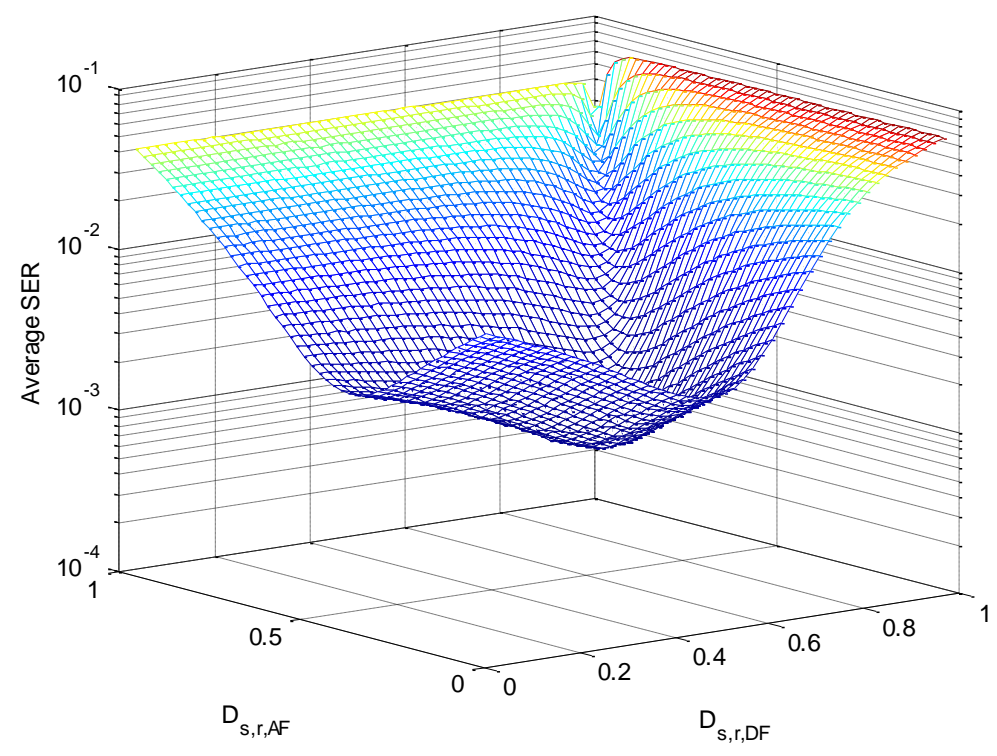

(a)

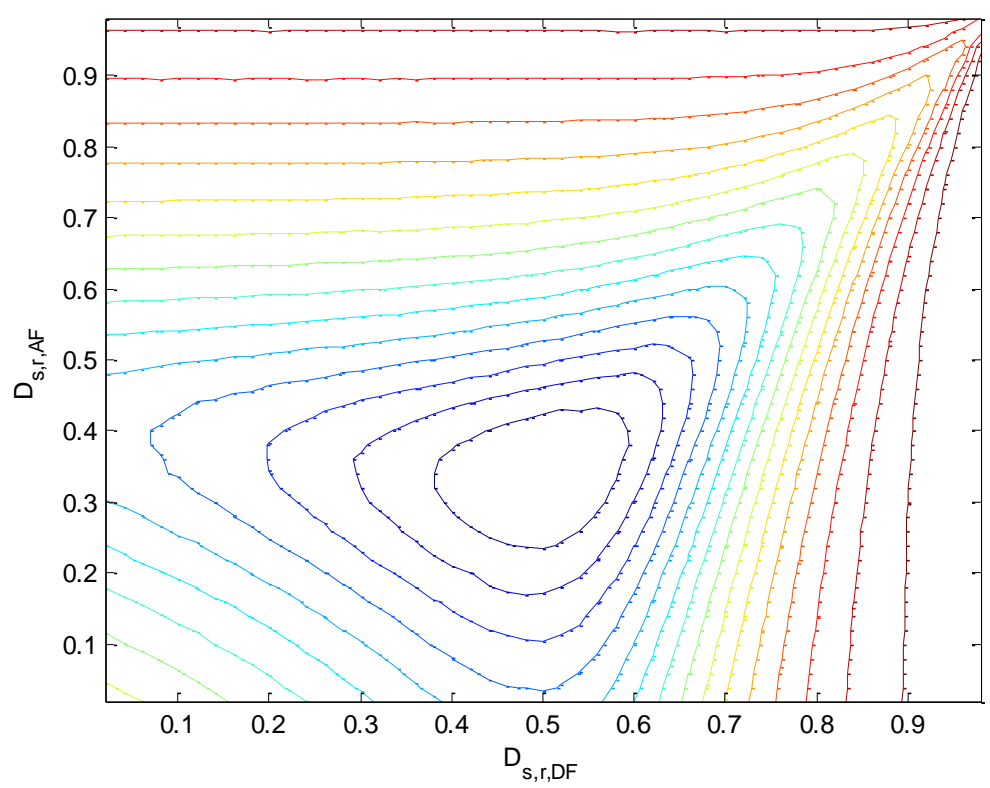

(b)

Figure 4. SER Depending on Various Relay Locations; Systems without Direct Link (a) SER Curve (b) SER Contour

Figures 3 and 4 represent SER curve and its corresponding contour for the system with direct link and without direct link, respectively. In the figures $D_{s, r, D F}$ and $D_{s, r, A F}$ represent the distance of s-r link using DF protocol and AF protocol, respectively. We let $\alpha=4$ and transmit energy at the source nod and the relay node as the same, i.e., $10 \mathrm{~dB}$. The results show that the relay location which provides minimum SER is different depending on the relaying protocol. For DF relaying protocol, optimum relay location is approximately 0.4 for both systems with and without direct link. For AF relaying protocol, the optimum location is the midpoint between the source node and the destination node for the system with direct link. The optimum location moves little bit toward to the 
destination node if the direct link is removed. Notice that the optimum performance cannot be achieved when two relay nodes are located at the same point, which is different from the system only with AF protocol or DF protocol in [3, 8-10]. However, the trend which provides optimum performance is the same when one relaying protocol is applied.

\subsection{The Effect of Location and Energy Allocation}

In this subsection we consider both location and energy allocation simultaneously. The location of relay node is allocated the same as previous section. For the energy allocation we constraint the total energy, and then the energy of source node and relay node is allocated. The total energy constraint can be expressed as

$$
\rho_{s}+\rho_{r}=\rho
$$

, where $\rho$ is total SNR, and the transmit SNR at the source node and the relay node is defined as

$$
\rho_{s}=\frac{\mathrm{E}_{s}}{N_{0}}
$$

and

$$
\rho_{r}=\frac{\mathrm{E}_{r}}{N_{0}}
$$

, respectively. Notice that at the previous subsection, we let the transmit energy the same, i.e., $\rho_{s}=\rho_{r}$. By combining eq. (10) and total energy constraint, the average received SNR at the relay and destination node can be express using transmitted SNRs as

$$
\begin{aligned}
& \bar{\gamma}_{s, r}=\rho_{s} \sigma_{s, r}^{2}=\rho_{s} D_{s, r}^{-\alpha}, \\
& \bar{\gamma}_{r, d}=\rho_{r} \sigma_{r, d}^{2}=\rho_{r} D_{r, d}^{-\alpha}, \\
& \bar{\gamma}_{s, d}=\rho_{s} \sigma_{s, d}^{2}=\rho_{s} D_{s, d}^{-\alpha} .
\end{aligned}
$$

For simulation, we let $\alpha=4$ and assume two relay nodes are collocated, then the location constraint is expressed as follows.

$$
\begin{aligned}
& D_{s, r, A F}=D_{s, r, D F}=D_{s, r}, \\
& D_{r, d, A F}=D_{r, d, D F}=D_{r, d}, \\
& D_{s, r}+D_{r, d}=D_{s, d}=1 .
\end{aligned}
$$

Based on the above notations, we let $\rho=15 \mathrm{~dB}$, and then we changed $\rho_{s}$ and $D_{s, r}$ simultaneously.

Figures 5 and 6 represent SER curve and its corresponding contour of the system with direct link and without direct link. Energy allocation is represented as ratio of transmit energy at the source node to the total energy. For the system with direct link the minimum SER can be achieved at the 0.4 location for the given energy allocation. We can see that the optimum location can be decided regardless of energy allocation. However, the Figure 5 reveals that the global optimum performance is achieved when lots of energy is allocated at the source node. The figure shows that the optimum performance is achieved when approximately 80 90\% of energy of total energy is assigned at the source node and the relay node is located about 0.4 location. 


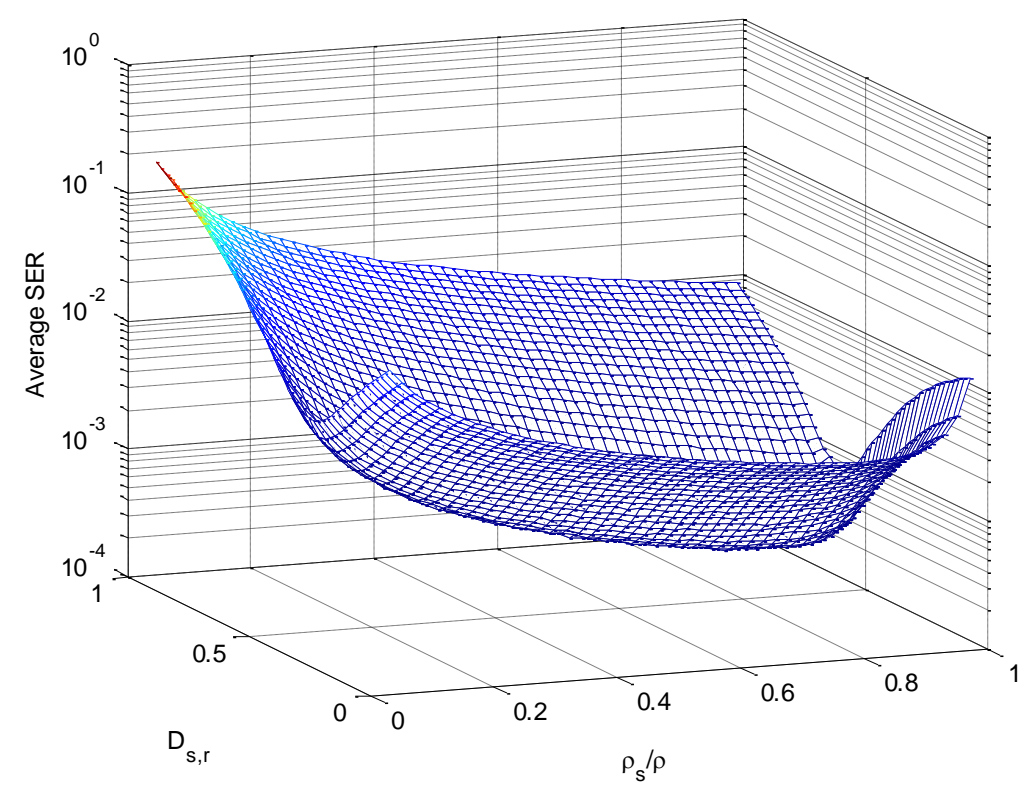

(a)

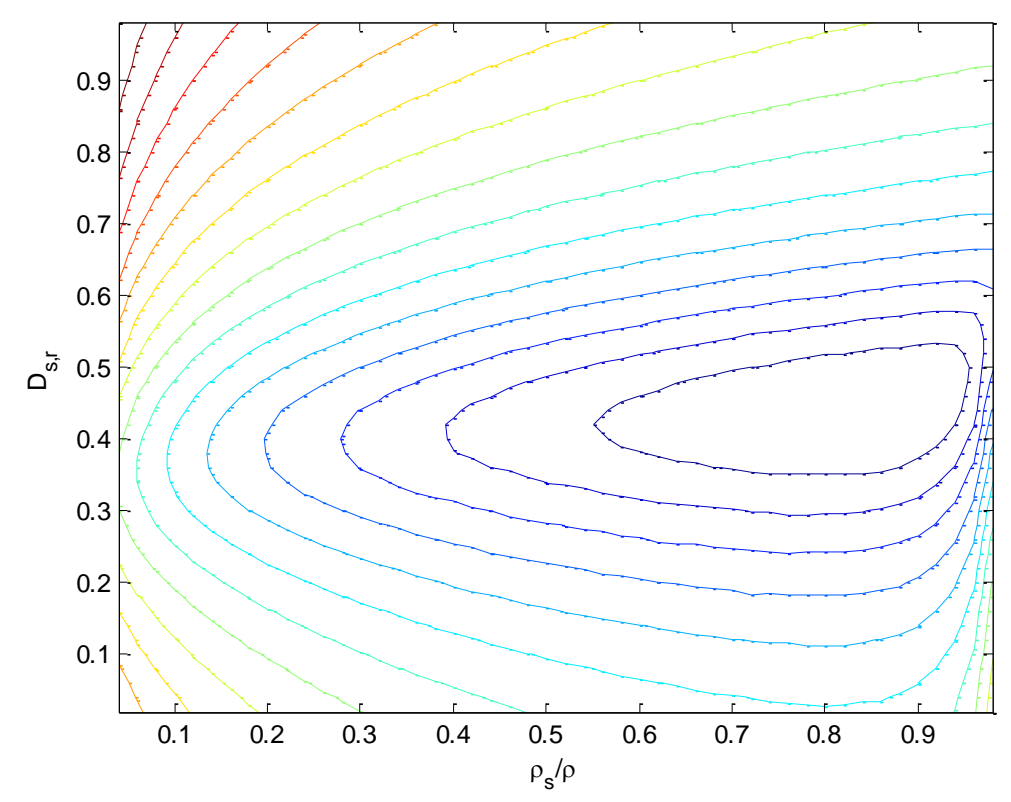

(b)

Figure 5. SER Depending on Various Relay Locations; Systems with Direct Link (a) SER Curve (b) SER Contour 


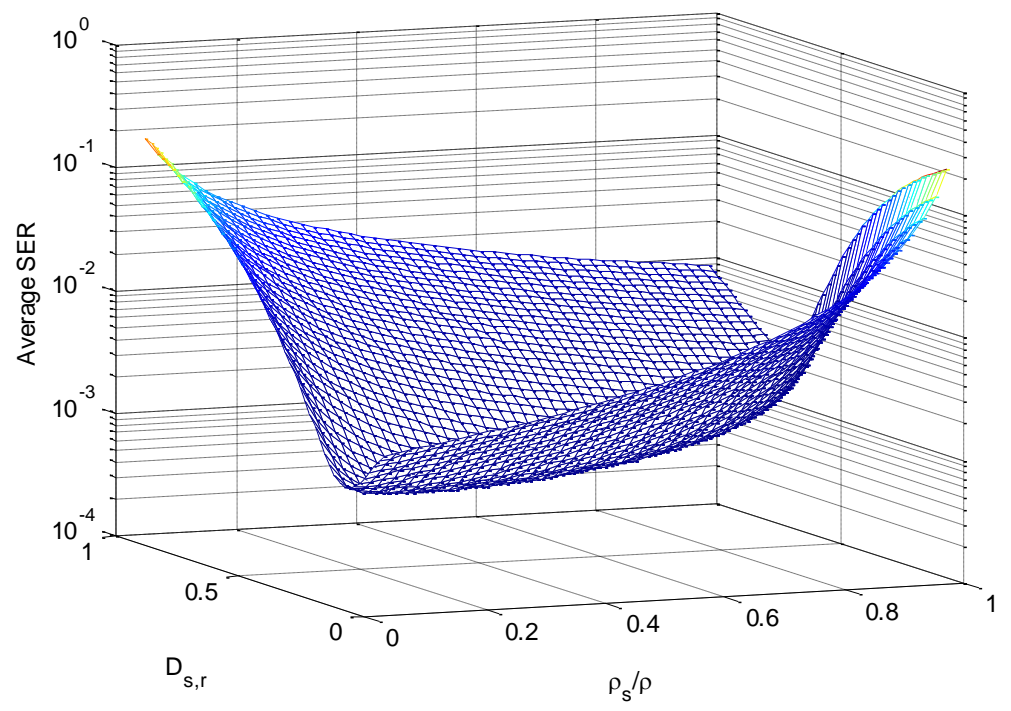

(a)

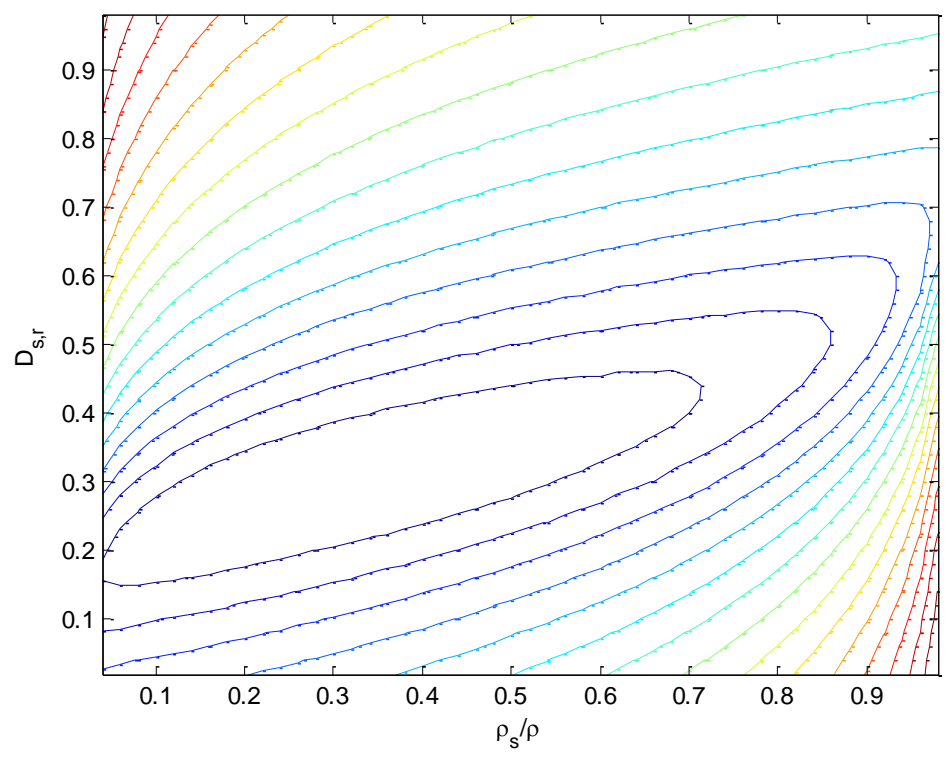

(b)

Figure 6. SER Depending on Various Relay Locations; Systems with Direct Link (a) SER Curve (b) SER Contour

Figure 6 represents the performance of the system without direct link. As we can see, the trend of optimum performance is different from the system with direct link. For the system without direct link the minimum SER point is changed depending on the energy allocation. Furthermore, the figure indicate that the global optimum point can be achieved when the relay node is located at the near source node and the energy of source node is allocated about $40 \%$ of the total energy. Therefore, the optimum point of the system with and without direct is somewhat different. Since the direct link affects the overall system performance, the energy allocation results of two systems are different. 


\section{Conclusions}

In this paper, we analyze the performance of two-relay networks with mixed relaying protocol in line topology. The performance of the proposed system was compared with existing systems. Then, the effect of relay location was analyzed depending on the existence of direct link. We reveal that there exists optimum location which provides minimum SER, and the results are different from the system using AF protocol or DF protocol only. Finally, we analyzed the performance of the system by considering the effect of relay location and energy allocation simultaneously. The results showed that the global optimum point is different depending on the existence of direct link.

\section{Acknowledgments}

This work was supported by the Jungwon University Research Grants (2016-34).

\section{References}

[1] J. N. Laneman and G. W. Wornell, "Energy-efficient Antenna Sharing and Relaying for Wireless Networks", Proceeding of Wireless Communication and Networking Conference, Chicago, IL, (2000) September 23-28.

[2] J. N. Laneman, D. N. C. Tse, and G. W. Wornell, "Cooperative Diversity in Wireless Networks: Efficient Protocols and Outage Behavior", IEEE Transactions on Information Theory, vol. 50, no. 12, (2004), pp. 3062-3080.

[3] A. Ribeiro, X. Cai, and G. B. Giannakis, "Symbol Error Probabilities for General Cooperative Links", IEEE Transactions on Wireless Communications, vol. 4, no. 3, (2005), pp. 1264-1273.

[4] D. Chen and J. N. Laneman, "Modulation and Demodulation for Cooperative Diversity in Wireless Sysetms", IEEE Transactions on Wireless Communications, vol. 5, no. 7, (2006), pp. 1785-1794

[5] Y. Lee, M-H. Tsai, and S-I Sou, "Performance of Decode-and-Forward Cooperative Communications with Multiple Dual-Hop Relays over Nakagami-m Fading Channels", IEEE Transactions on Wireless Communications, vol. 8, no. 6, (2009), pp. 2853-2859.

[6] S. S. Ikki and M. H. Ahmed, "Performance Analysis of Adaptive Decode-and-Forward Cooperative Diversity Networks with Best-Relay Selection”, IEEE Transactions on Communications, vol. 58, no. 1, (2010), pp. 68-72.

[7] H. Li and Q. Zhao, "Distributed Modulation for Cooperative Wireless Communications", IEEE Signal Processing Magazine, vol. 23, no. 5, (2006), pp. 30-36.

[8] W. Cho, L. Yang, "Optimum resource allocation for relay networks with differential modulation", IEEE Transactions on Communications, vol. 56, no. 4, (2008), pp. 531-534.

[9] W. Cho, R. Cao, L. Yang, "Optimum resource allocation for amplify-and-forward relay networks with differential modulation”, IEEE Transaction on Signal Processing, vol. 56, no. 11, (2008), pp.5680-5691.

[10] R. Cao, L. Yang "The affecting factors in resource optimization for cooperative communications: A case study", IEEE Transaction on Wireless communications, vol. 11, no. 12, (2012), pp.4351-4361.

[11] H. Halabian, A. Zainaldin, and I. Lambadaris, "Optimal Joint Resource Allocation and Power Control in Bidirectional Relaying Networks" IEEE Transactions on Vehicular Technology, vol. 63, no. 9, (2014), pp. 4520-4535.

[12] M. Liem, V. B. Mendiratta, "Mission critical communication networks for railways", Bell Labs Technical Journal, vol. 16, no. 3, (2011), pp. 29-46.

[13] J. Calle-Sanchez, M. Molina-Garcia, J. I. Alonso, A. Fernandez-Duran, "Long Term Evolution in high speed railway environments: feasibility and challenges", Bell Labs Technical Journal, vol. 18, no. 2, (2013), pp. 237-253.

[14] 3GPP TR 22.803, “Feasibility study for proximity services (ProSe)" (2013).

[15] J. You, Z. Zhong, S. Yang, Y. Liu, G. Wang, "Performance analysis of optimal relay selective cooperation in railway network", IET International Conference on Wireless, Mobile and Multimedia Networks", pp. 315-319, Beijing, (2013), November 22-25.

[16] J. Calle-Sanchez, E. Martinez-de-Rioja, M. Molina-Garcia, J. I. Alonso, "Performance of LTE mobile relay node usage for uplink access in high speed railway scenarios", in IEEE Vehicular Technology Conf. Spring, pp. 1-5, Glasgow, Scotland, (2015), May 11-14. 


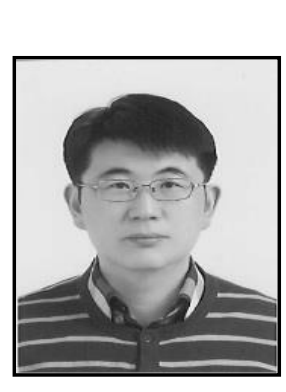

\begin{abstract}
Authors
Woong Cho, received the B.S. degree in electronics engineering from the University of Ulsan, Ulsan, South Korea, in 1997, the M.S. degrees in electronic communications engineering and electrical engineering from Hanyang University, Seoul, South Korea, in 1999, and the University of Southern California, Los Angeles, in 2003, respectively, and the Ph.D. degree in electrical and computer engineering from University of Florida, Gainesville, in 2007. He was a senior research engineer from Feb. 2008 to Feb. 2012 in Electronics and Telecommunications Research Institute (ETRI), Daejeon, South Korea. He is currently with the department of Computer System Engineering in Jungwon University, Goesan, South Korea. His research interests are cooperative/relay networks, vehicular communications, and signal processing for wireless communications.
\end{abstract}


International Journal of Control and Automation Vol.10, No.4 (2017) 